\title{
Incorporating financial protection into decision rules for publicly financed healthcare treatments
}

\author{
Peter C. Smith \\ Imperial College Business School \& Centre for Health Policy \\ Exhibition Road \\ London SW7 2AZ \\ United Kingdom \\ Tel: +44 (0)207594 1904 \\ e-mail: peter.smith@imperial.ac.uk
}

December 2011

Keywords: public healthcare; financial protection; health benefits package

\section{Acknowledgements}

This material has been presented in a number of settings, including seminars at the Wharton Business School, the University of Leuven, the University of Brescia, City University, Erasmus University, the University of Bergen and the London School of Economics. I am grateful to participants, the journal referees and to my colleague Rodrigo Moreno-Serra for comments. 


\title{
Incorporating financial protection into decision rules for publicly financed healthcare treatments
}

\begin{abstract}
Almost all health systems seek to offer some form of publicly financed healthcare insurance, and governments must therefore choose the size of the benefits package and the types of treatments to cover. Conventionally, the usual approach recommended by economists has been to recommend choices based on cost-effectiveness of treatments, using metrics such as the 'cost per quality adjusted life year'. However, this approach is based on assumption of health maximization subject to a budget constraint, and ignores the potential impact of any additional concern with protecting individuals from the financial consequences of a health shock. Furthermore, it does not take account of the possible availability of complementary privately funded health care. This paper develops a model in which risk-averse individuals care about health, but also place a value on protection from the financial consequences of rare but costly events. The paper shows how conventional cost-effectiveness analysis can readily be augmented to take account of financial protection objectives. The results depend on whether or not there exists a market in complementary privately funded healthcare. They have important implications for the methodology adopted by health technology assessment agencies, and for the broader design of publicly funded health systems.
\end{abstract}




\section{Incorporating financial protection into decision rules for publicly financed healthcare treatments}

\section{INTRODUCTION}

The principal means of financing most mature health systems is a statutory health care insurance scheme, covering all citizens whatever their circumstances, in what has become known as universal health coverage (Schreyögg, Stargardt et al. 2005). The statutory system is funded by some sort of taxation or social insurance, with contributions usually according to some measure of income, and unrelated to health status. In many countries, the statutory package of care is supplemented by a private health care market, funded either by out-ofpocket payments or by voluntary health insurance, with financial contributions reflecting actual or expected use of services (Mossialos and Thomson 2004).

Most wealthy countries seek to make the statutory package reasonably comprehensive, ensuring that all citizens are insured for reimbursement of most mainstream health care (albeit sometimes with a modest user copayment). However, health care is offering rapidly increasing opportunities to address sickness and disability, citizens are placing increasing demands on their health care systems, and public sources of funds are coming under increasing strain. There may be limits to the extent to which the traditional sources of public finance for statutory insurance can be exploited. The principle of comprehensiveness is therefore coming under strain. In rich countries, the policy questions therefore arise: should some interventions be removed from the statutory package; and if so, which ones?

In low income countries, there are often limited financial resources available for statutory insurance, based on a slender tax base and (sometimes) donor funds. There is therefore usually no attempt to offer comprehensive coverage, and a much heavier reliance is placed on personal finance of health care, usually in the form of out-of-pocket payments (Gertler and Van der Gaag 1990). A key policy question in these circumstances is the extent to which the limited statutory system is being deployed to best effect. In many countries there has been a persistent concern that the statutory funds available are being spent on interventions that are not cost-effective, or are not supporting those most in need, namely the sick and the poor (Hauck, Smith et al. 2002). 
Such concerns have led to an increasingly concerted effort in all types of health system to specify explicitly an 'essential' package of health care that is covered by the statutory insurance fund (Jost 2005). The intention is to create a set of interventions to which all qualifying citizens have an entitlement when clinical indications are satisfied. The usual assumption is that receipt of care will be free or subject to a small copayment. Of course, the scope of the essential package is constrained by the financial resources available to the statutory scheme.

Economists have championed the use of the cost-effectiveness ratio as the main criterion for selecting interventions for inclusion in the essential package of care (Drummond, Sculpher et al. 2005). This policy prescription flows from the notion of maximizing health benefits subject to a public finance budget constraint. Cost-effectiveness analysis may therefore be relevant at the margin for choosing interventions to exclude from a near-comprehensive statutory package of health care. However, the cost-effectiveness criterion may on its own be inappropriate for determining the essential package when either equity concerns or private payments play a significant role in funding health care (Smith 2005). Furthermore, the conventional cost-effectiveness approach takes no account of any policy concern with protecting individuals from the financial consequences of health shocks. Indeed, the World Health Report 2010 highlights the key role played by financial protection objectives in shaping many countries’ policies towards publicly financed healthcare.

The purpose of this paper is therefore to identify the optimality criteria that apply to publicly financed healthcare when there is a policy concern with financial protection as well as health maximization. Furthermore the paper seeks to generalize previous work by examining the implications of different assumptions about the nature of the complementary private market for health services. A further contribution of the paper is to model optimal choices when the epidemiology of disease (and therefore the need for treatment) differs between wealth groups and there is a concern with equity.

The paper is structured as follows. The next section briefly discusses the relevant academic literature on optimality conditions for the size and content of a statutory package of health insurance. A stylized model of healthcare is introduced, in which individuals have access to a statutory package of healthcare, but might augment it in the form of out-of-pocket payments 
or complementary private health insurance. The paper then examines the government's problem, which is to design an optimal statutory package of insured health care funded from public finances, given the known responses of citizens. The paper ends with policy conclusions.

\section{THE SIZE AND CONTENT OF THE STATUTORY PACKAGE}

There is an economic argument that the prime role of mandatory health insurance is to effect a redistribution of wealth from rich to poor, by enabling poor people to secure health insurance that they would not otherwise be able to afford. Some might argue that it - if such a transfer is desired - a more efficient policy than mandatory health insurance would be to implement an equivalent wealth redistribution (through the tax system) and allow consumers to choose the level and content of their insurance package from a competitive market. The imposition of a statutory package merely constrains consumers from making their preferred arrangements.

The arguments against such a position are manifest, and include:

- Market failures in the putative private insurance market, including information weaknesses, cream skimming and adverse selection

- High transaction costs associated with private insurance markets

- Moral hazard, in the sense that some citizens may choose not to insure in the belief that society will nevertheless offer healthcare if they fall sick (in effect, there may be an implicit 'safety net' package of care available to all)

- Health care may be a merit good, and it may therefore be easier politically to effect the transfer to the poor through provision of benefits in kind (in the form of health insurance) than cash transfers.

The fact that almost all developed countries and an increasing number of lower income countries have adopted some sort of mandatory package of health care indicates that these and other arguments outweigh the prescriptions of a conventional economic efficiency argument. This paper therefore takes as given the need to specify a mandatory package of care, and seeks to guide policy makers on the size and nature of this publicly funded 'health basket’ (Schreyögg, Stargardt et al. 2005). 
It is important to note that the paper makes no judgement on the precise details of how funding of the statutory package of care is arranged. This might be through local government, national government, employer-based social insurance, or competitive social insurance. The only requirements are that the insurance arrangement should be mandatory (and therefore effectively publicly funded), and that the minimum contents of the package to be insured should be specified. Furthermore, I shall assume that premiums must be unrelated to sickness levels (community rating). These assumptions do not preclude, for example, a competitive market in insurers who compete on price or supplementary contents of the insurance package.

In contrast to developed nations, many low income countries have no viable market in private health insurance. Instead, they rely heavily on user charges as a source of finance, and are frequently unable to protect citizens from catastrophic health care payments (McPake 1993; Mwabu 2001). There are numerous adverse consequences of this lack of financial protection, from both a macroeconomic and micro perspective (World Health Organization 2010). User charges also have a disproportionately adverse impact on poor people (Castro-Leal, Dayton et al. 2000). In principle, therefore, even in the absence of any statutory health insurance, some form of private health insurance might offer a Pareto improvement over a system of direct user charges. However, there are in practice often no trusted institutions in place to manage any insurance function in low income countries. Worldwide, pre-payed private insurance accounts for little more than 5\% of all health expenditure (World Health Organization 2010). In the absence of an insurance function, either governmental or non-governmental, citizens must either forego health care, or resort to direct out-of-pocket payments.

There is a surprising shortage of relevant theoretical literature on the interaction between user charges, voluntary health insurance and the public package of health care. Smith (2005) presents a theoretical model of 'optimal' user charges for specific treatments when there is a limit to the size of the statutory package. Hoel (2007) introduces diminishing marginal utility of wealth to determine which treatments should be included in a budget-constrained statutory package when the only alternative financing is through private out-of-pocket payments.

Private health insurance (alongside a statutory package of care) can take three broad forms: substitutive, supplementary or complementary (Mossialos and Thomson 2004). Substitutive insurance is purchased as an alternative to the statutory insurance, with the implication that 
those who elect to take out such coverage are at least partially exempt from the premiums or taxes associated with the statutory package.

Supplementary private insurance covers services in the statutory package, but insurees receive no exemption from payments towards the statutory package, and therefore enjoy double coverage. A market in supplementary insurance implies that it must offer a perceived quality advantage over the care secured by the statutory package, perhaps in the form of reduced waiting times or access to superior facilities. In contrast, complementary insurance offers full or partial cover for services that are excluded or not fully covered by the statutory health care system. In particular, as in France, it may cover liability for copayments levied on services within the statutory package.

There has been a small economic literature on the role of voluntary health insurance alongside a publicly-funded 'essential' package of health care. Besley (1989) examines the extent to which the problem of moral hazard can be abated by augmenting a competitive insurance market with publicly funded catastrophic health insurance. The paper stimulated a lively academic exchange that is not strictly relevant to the problem addressed in this paper, but which does highlight the complexity of formulating mathematical models in this domain, and the need for clarity about the assumptions underlying any modelling (Selden 1993; Blomqvist and Johansson 1997).

Petretto (1999) examines the functioning of a publicly insured essential package of care alongside a market in private complementary insurance. The citizen is free to choose the insured copayment rate for the complementary services. Citizens make three contributions to health care financing: a tax contribution; a private insurance premium; and the residual copayment. A form of optimal income taxation model is used to analyse the government's problem, which is to select the optimal statutory copayment rate, in the light of response in the private insurance market. Smith (2006) develops a theoretical model of the optimal statutory package in the presence of complementary voluntary health insurance.

There is also a broader public economics literature on the public/private mix. Blackorby and Donaldson (1988) note that the sort of 'in kind' transfers implied by public insurance may be preferred to cash transfers when (as in the case of health care) they are non-tradeable. In contrast to cash transfers, in-kind transfers can ensure that only the intended beneficiaries 
receive the relevant service. Munro (1991) examines the implications for optimal taxation policy of such transfers. Ireland (1990) models the integration of in-kind transfers and cash transfers, in the form of unconditional payments to the poor, and conditional subsidies of private consumption, for example in the form of vouchers.

Epple and Romano (Epple and Romano 1996) examine the public/private mix within a majority voting model, and find that society's choice may depend on the balance of electoral power between middle income voters (who prefer higher public provision) and a coalition of high and low earners (who prefer lower public provision). Finally, Besley and Coate (1991) note the crucial redistributive function of social provision of private goods. Providing the quality of the social good is not 'too high', some richer households will - without the need for financial compensation - opt out of the social good in order to consume its private counterpart, yielding an implicit financial transfer to the poor. In a similar vein, Blomqvist and Horn (1984) examine the transfer from the healthy to the sick implicit in a system of statutory insurance in a health care setting.

Little of this literature models the great heterogeneity of services that make up healthcare, or the variations in epidemiology across social groups. It focuses principally on the choice of taxation and copayment rates, and does not address a fundamental concern of policy-makers - which types of service to include in the essential package. In contrast, this paper generalizes the work of Smith (2006) and Hoel (2007) to examine the choice of the statutory package when the alternative to public provision is private payments, in the form of either out-ofpocket payments or voluntary private insurance.

\section{THE MODEL}

The approach used in this paper is to model optimal government choices in the light of known individual responses to health insurance policy. The individual is risk averse, and cares about health and wealth. The government seeks to maximize a social welfare function that incorporates utility and equity concerns. The model therefore incorporates differences between wealth groups in (a) underlying health and (b) epidemiology (probability of disease). This leads to variations in treatment choices between wealth groups. However, it is not necessary to model variability within wealth groups. 
There is a set of $n$ health care problems, and for each problem there is a technology $i$ available at a known constant price $x_{i}$. The technologies are assumed to be efficient, in the sense that no technology is dominated by any other for the specified condition (these are the most cost-effective technologies for each condition). Additional assumptions that are made are (a) the treatments are additively separable - there are no interactions between diseases or treatments (b) the need for treatment $i$ in wealth group $y$ occurs with probability $\pi_{i}(y)$ (though it may not always necessarily be poorer people who have higher incidence for all diseases) (c) the expected cost of each treatment (probability times $x_{i}$ summed across all wealth groups) is small relative to total expected healthcare expenditure and (d) the health benefits $h_{i}$ of any treatment are equal for all groups.

\section{The individual}

Adapting the notation of Hoel (2007), I assume that an individual's utility is separable in wealth and health. If a health problem occurs so that treatment $i$ is needed, there is without treatment a known loss of individual utility equal to $h_{i}$ that does not differ between individuals, which we can think of as the health-related gain of treatment. Without loss of generality, it can be assumed that treatment returns an individual to full health. Thus, in the absence of any payment for treatment, utility of an individual with wealth $y$ is $u(y)$ and without treatment it is $u(y)-h_{i}$. The wealth- related utility function $u($.$) obeys the usual$ assumptions of diminishing marginal utility. This formulation allows the possibility of incorporating into the function $u$ (.) different underlying health (such as life expectancy independent of healthcare). However, a limiting assumption in this analysis is that healthcarerelated utility $h$ is independent of wealth $y$.

In the main text I consider a dichotomy of just 'rich' and 'poor' people, designated $R$ and $P$, distributed in the population in proportions $\rho$ and $(1-\rho)$. The continuous wealth measure $y$ is therefore replaced by a social group indicator $Z$. The initial wealth of group $\mathrm{Z}$ is indicated by $y_{0}^{Z}$, and the expected incidence of disease $i$ by $\pi_{i}^{Z}$, with the aggregate incidence equal to $\Pi_{i}=\rho \pi_{i}^{R}+(1-\rho) \pi_{i}^{P}$. The implications of a larger number of wealth categories or a continuous distribution of wealth do not materially affect the results, and are treated briefly in the appendix. 
With no statutory health care package, individuals must finance all care privately. Assume first that there are no insurance arrangements of any sort, and that the individual must finance the treatment out-of-pocket when the need arises. Then, conditional on the need for treatment $i$ arising, the health-related gain of treatment is $h_{i}$ and loss in wealth-related utility $u\left(y-x_{i}\right)$. So the treatment will be purchased if $h_{i}+u\left(y-x_{i}\right)>u(y)$. For a treatment yielding a health gain $h$ there is therefore for each social group $Z$ a reservation price $x_{Z}^{*}$ at which an individual will be indifferent between receiving and rejecting the treatment, such that $h=u\left(y_{0}^{Z}\right)-u\left(y_{0}^{Z}-x_{Z}^{*}\right)$ - the potential health gain equals the loss of wealth-related utility associated with paying for the treatment.

Figure 1 illustrates the locus of pairs $(x, h)$ at which such indifference occurs for the rich and the poor wealth group. To the left of the curve, treatment will be purchased, to the right it will be rejected. The space of acceptable treatments enjoyed by the poor group is of course smaller than the equivalent space for the rich group, reflecting its higher marginal utility of wealth. The curvature of the locus reflects the diminishing marginal utility of wealth (its gradient is $\partial u / \partial y$ at the post-treatment level of wealth). Treatments with identical cost effectiveness measures $x / h$ might be accepted if they entail low expenditure (and low health gain), whilst rejected if they require higher expenditure (even though they yield the same proportionate increase in health gain).

$<$ Figure 1 about here $>$

Consider now the case when there is still no statutory insurance, but individuals are able to purchase private health insurance at an actuarially fair price. For the treatments included in the insurance package, this replaces uncertain ex post payments (or denial of treatment) with a certain ex ante payment, leading to a welfare gain for risk averse individuals. For an individual in wealth group $Z$ the optimal insurance package is identified by maximizing expected utility:

Maximize $\sum_{i}\left(\pi_{i}^{Z} \theta_{i}^{Z} h_{i}\right)+u\left(y_{0}^{Z}-\sum \pi_{i}^{Z} \theta_{i}^{Z} x_{i}\right)$ 
where the decision variables $\left\{\theta_{i}^{z}\right\}_{i=1}^{n}$ are binary variables indicating whether or not insurance for the treatment will be purchased by someone in group $Z$. The amount $\sum_{i} \pi_{i}^{Z} \theta_{i}^{Z} x_{i}$ indicates the premium associated with the chosen insurance package. This yields the rule that intervention $i$ is included if and only if its cost effectiveness exceeds a threshold: $\frac{h_{i}}{x_{i}} \geq \frac{\partial u^{Z}}{\partial y}$.

where the marginal conditions apply at the wealth of group $Z$ after the relevant premium has been paid. That is, the insurance package is based solely on the cost-effectiveness of each intervention. Under the assumption of diminishing marginal utility of wealth, the threshold decreases with wealth, yielding the obvious result that the rich insure for a more extensive healthcare package than the poor.

Voluntary insurance shifts the burden of paying for health treatments from out-of-pocket to insurance. Its impact on access to health care is illustrated in Figure 2. The person's original 'out-of-pocket' curve of indifference (between treatment and non-treatment) is shifted from $\mathrm{OA}$ to $\mathrm{OB}$, because of the wealth loss associated with the payment of the insurance premium. However, the insurance chosen by the individual means that he or she can now receive treatment whenever the cost-effectiveness of the treatment lies on or above the line OC, with the slope $\partial u / \partial y$ of the indifference curve OB at the origin (the post-premium marginal utility of wealth, in line with the first order condition noted above). Notice that the individual now secures access to some high cost, high benefit treatments that would have been not purchased if payment had been out-of-pocket, indicated by the shaded area between OC and OA. However, the reduced wealth associated with paying the premium leads to loss of access to some treatments with modest cost-effectiveness, indicated by the unshaded area between OC and $\mathrm{OA}$ at lower levels of expenditure.

$<$ Figure 2 about here $>$

Now consider the individual's private healthcare decision when there is a statutory package of health services, funded from public finances. I assume that the quality of publicly and privately purchased treatments are identical, so there is no incentive to purchase privately any treatment that is in the statutory package. The individual must therefore decide whether or not 
to purchase privately some treatments not contained in the statutory benefits package. If private insurance is not available, then this decision is taken only when sickness arises, and is modelled simply by adapting the out-of-pocket decision discussed above to the situation in which some treatments are covered by the statutory package. A treatment not in the package will be purchased out-of-pocket if $h_{i}>u\left(y_{0}^{Z}-t^{Z}\right)-u\left(y_{0}^{Z}-x_{i}-t^{Z}\right)$, where $t^{Z}$ is the tax levied on wealth group $Z$ as a contribution to funding the public package.

If private insurance is available, the decision as to whether or not to insure can be modelled by comparing expected utility (a) under the mandatory package only, when utility will be a function of the expected benefits of the mandatory package and its personal tax cost; and (b) supplementing the mandatory package with some private healthcare, when utility will be a function of the expected benefits of the combined private and statutory packages, and the personal tax cost plus private payments.

The individual makes the choice of private insurance in the knowledge of the government's chosen package and the associated tax payments needed to finance the package. The status of intervention $i$ in the statutory package chosen by the government is indicated by a binary variable $\lambda_{i}$, where $\lambda_{i}=1$ if intervention $i$ is in the statutory package, $\lambda_{i}=0$ otherwise. Individuals may purchase private healthcare insurance, covering interventions not included in the statutory package $\lambda$. As above, the extent of the private package for wealth group $Z$ is indicated by the binary choice variables $\left\{\theta_{i}^{Z}\right\}$. An individual in wealth group $Z$ will then choose a complementary package $\left\{\theta_{i}^{Z}\right\}_{i=1}^{n}$ so as to:

$$
\begin{array}{ll}
\text { Maximize } & \sum_{i}\left(\pi_{i}^{z}\left(\theta_{i}^{Z}+\lambda_{i}\right) h_{i}\right)+u\left(y_{0}^{z}-t^{z}-\sum_{i} \pi_{i}^{z} \theta_{i}^{Z} x_{i}\right) \\
\text { subject to } & \theta_{i}^{Z}+\lambda_{i} \leq 1 \quad \forall i
\end{array}
$$

If private insurance is purchased (some $\theta_{i}^{Z}=1$ ) then the optimality conditions for the selected interventions are:

$$
\frac{h_{i}}{x_{i}} \geq \frac{\partial u^{Z}}{\partial y}
$$

where the conditions apply at the wealth of group $Z$ after the relevant premium and taxation have been paid, and there is equality for the marginal intervention. In general, an increase in 
the scope of the statutory package will reduce the wealth of all citizens (through the necessary tax contributions $t^{Z}$ ), thereby in general increasing the threshold for inclusion in the private insurance package (and reducing the size of it).

Note that the chosen set of private treatments under different mandatory packages in effect specify a reaction function for group $Z$ to any government policy. That is, the vector $\boldsymbol{\theta}^{Z}=\mathbf{r}^{Z}\left(\boldsymbol{\lambda}, t^{Z}\right)$ indicates the response in the voluntary insurance market of social group $Z$ to mandatory package $\lambda$ and tax $t^{Z}$. The set of response functions acts as a constraint in the government's optimization problem.

\section{The Government}

Having decided to mandate a statutory package of healthcare, the government must decide on the extent of the package, the decision rules for including treatments in the package, and how the package is to be financed. I assume that there is a social welfare function $W\left(\rho U^{R},(1-\rho) U^{P}\right)$ that the government wishes to maximize, subject to the constraint that the costs of the chosen statutory package must be funded by tax payments by all citizens. If a treatment is included in the package, it is universal - all citizens are entitled to that treatment when appropriate.

First assume that there is no private health care of any sort. Then the government's problem is to choose interventions $\left\{\lambda_{i}\right\}_{i=1}^{n}$ and taxes $t^{R}$ and $t^{P}$ for the rich and poor so as to:

$$
\begin{array}{ll}
\text { Maximize } & W\left(\rho\left[\sum_{i} \pi_{i}^{R} \lambda_{i} h_{i}+u\left(y_{0}^{R}-t^{R}\right)\right],(1-\rho)\left[\sum_{i} \pi_{i}^{P} \lambda_{i} h_{i}+u\left(y_{0}^{P}-t^{P}\right)\right]\right) \\
\text { Subject to } & \sum_{i} \lambda_{i}\left\{\rho \pi_{i}^{R}+(1-\rho) \pi_{i}^{P}\right\} x_{i}=\rho t^{R}+(1-\rho) t^{P} \\
& \lambda_{i} \in\{0,1\}
\end{array}
$$

First order conditions yield the result that intervention $i$ is selected if and only if:

$$
\frac{h_{i}}{x_{i}} \geq \frac{\mu\left[\rho \pi_{i}^{R}+(1-\rho) \pi_{i}^{P}\right]}{\left[\rho \pi_{i}^{R} \frac{\partial W}{\partial U^{R}}+(1-\rho) \pi_{i}^{P} \frac{\partial W}{\partial U^{P}}\right]}=\frac{\mu \Pi_{i}}{\left.\mid \rho \beta_{R} \pi_{i}^{R}+(1-\rho) \beta_{P} \pi_{i}^{P}\right]}
$$


where $\mu$ is the opportunity cost of tax funds and $\beta_{Z}=\frac{\partial W}{\partial U^{Z}}$ is the marginal social value of an improvement in utility for group $Z$. This equation effectively adjusts $\mu$ for variations in the social importance of the two population groups, reducing the hurdle rate for interventions with a high incidence in the poorer group if there is a pro-poor equity concern. It is consistent with the policy recommendation of adjusting the cost-effectiveness ratios of clinical interventions according to their equity implications (Williams, Tsuchiya et al. 2005).

The tax contributions for each social group $Z$ satisfy the marginal conditions:

$$
\frac{\partial W}{\partial U^{Z}} \frac{\partial u^{Z}}{\partial y}=\mu \text { or } \frac{\partial u^{Z}}{\partial y}=\mu / \beta_{Z}
$$

where the derivatives are evaluated at post-tax levels of wealth $\left(y_{0}^{Z}-t^{Z}\right)$. A crucial role of the tax payments is therefore to equalize social marginal utility of wealth across social groups. The special case of a linear wealth tax constrains the government's options for effecting transfers, and the marginality condition becomes:

$\mu=\left(\rho \frac{\partial W}{\partial U^{R}} \frac{\partial u^{R}}{\partial y} y_{0}^{R}+(1-\rho) \frac{\partial W}{\partial U^{P}} \frac{\partial u^{P}}{\partial y} y_{0}^{P}\right) /\left(\rho y_{0}^{R}+(1-\rho) y_{0}^{P}\right)$

or for many social groups:

$\mu=\sum_{Z} \rho_{Z} \frac{\partial W}{\partial U^{Z}} \frac{\partial u^{Z}}{\partial y} y_{0}^{Z} / \sum_{Z} \rho_{Z} y_{0}^{Z}$

Suppose now that out-of-pocket complementary private healthcare is available. If neither wealth group chooses to use private healthcare, the situation remains as just examined (no private care). If both groups choose to use some private care not in the public package, the marginal conditions are those discussed earlier with no statutory insurance, although note there will have been an effective cash transfer between groups brought about by the tax contributions for the statutory insurance. However, the analytically interesting case is when the rich group chooses to pay out-of-pocket for private healthcare not covered by the statutory insurance while the poor group does not.

It is worth noting here the implications of including treatment $i$ in the statutory package:

a) for a poor person, the procedure becomes available, so there is an expected health gain $\pi_{i}^{P} h_{i}$; 
b) for a rich person, there is no need to purchase the treatment out-of-pocket at price $x_{i}$ with probability $\pi_{i}^{R}$;

c) for both groups, there is an increase in the tax payment, in sum equal to $\Pi_{i} x_{i}$.

For the marginal procedure, the welfare gains associated with (a) and (b) will be balanced against the wealth losses represented by (c).

Because the rich would pay out-of-pocket anyway for treatments not included in the package, the direct impact of the statutory package is now on their wealth (not their health), and the government's optimization problem becomes:

$$
\begin{array}{ll}
\text { Maximize } & W\left(\rho\left[\sum_{i} \pi_{i}^{R}\left(\lambda_{i} u\left(y_{0}^{R}-t^{R}\right)+\left(1-\lambda_{i}\right) u\left(y_{0}^{R}-t^{R}-x_{i}\right)\right)\right],(1-\rho)\left[\sum_{i} \pi_{i}^{P} \lambda_{i} h_{i}+u\left(y_{0}^{P}-t^{P}\right)\right]\right) \\
\text { Subject to } & \sum_{i} \lambda_{i}\left\{\rho \pi_{i}^{R}+(1-\rho) \pi_{i}^{P}\right\} x_{i}=\rho t^{R}+(1-\rho) t^{P} \\
& \lambda_{i} \in\{0,1\}
\end{array}
$$

The solution for an intervention to be included in the statutory package is therefore:

$$
\rho \pi_{i}^{R} \beta_{R}\left[u\left(y_{0}^{R}-t^{R}\right)-u\left(y_{o}^{R}-t^{R}-x_{i}\right)\right]+(1-\rho) \pi_{i}^{P} \beta_{P} h_{i} \geq \mu \Pi_{i} x_{i}
$$

with equality for the marginal intervention. On the left hand side, the first expression gives the financial benefits to the rich of not needing to pay out of pocket for intervention $i$ if the need arises. The second expression represents the health benefits to the poor of including intervention $i$ in the statutory package. The right hand side gives the incremental tax cost to both rich and poor of including intervention $i$ in the statutory package.

Notice that this rule is analogous to the more conventional cost-effectiveness criterion for inclusion in the package, which ranks treatments according to the ratio $h_{i} / x_{i}$. The difference is that wealth benefits $\left[u\left(y_{0}^{R}-t^{R}\right)-u\left(y_{0}^{R}-t^{R}-x_{i}\right)\right\rfloor$ replacing health benefits $h_{i}$ for richer groups who take advantage of private healthcare if the treatment is not included in the statutory package. By definition, this expression is less than $h_{i}$. Relative to the conventional ranking, this new rule will therefore favour treatments for diseases of poor people. Furthermore, for treatments with the same cost-effectiveness rates $h_{i} / x_{i}$, it will favour high cost treatments, for which the loss of wealth-related utility $\left[u\left(y_{0}^{R}-t^{R}\right)-u\left(y_{0}^{R}-t^{R}-x_{i}\right)\right\rfloor / x_{i}$ would be high relative to lower cost treatments with identical ratio $h_{i} / x_{i}$. 
When complementary private insurance is available, the rich now insure some treatments, and do not have to rely on out-of-pocket payments for treatments outside the statutory package. Assume that the rich choose to insure a package of treatments $\left\{\theta_{i}^{R}\right\}_{i=1}^{n}$. The government's optimization problem is revised to the following:

$$
\begin{array}{ll}
\text { Maximize } & W\left(\rho\left[\sum_{i} \pi_{i}^{R}\left(\lambda_{i}+\theta_{i}^{R}\right) h_{i}+u\left(y_{0}^{R}-t^{R}-\sum_{i} \pi_{i}^{R} \theta_{i}^{R} x_{i}\right)\right],(1-\rho)\left[\sum_{i} \pi_{i}^{P} \lambda_{i} h_{i}+u\left(y_{0}^{P}-t^{P}\right)\right]\right) \\
\text { Subject to } & \sum_{i} \lambda_{i}\left\{\rho \pi_{i}^{R}+(1-\rho) \pi_{i}^{P}\right\} x_{i}=\rho t^{R}+(1-\rho) t^{P} \\
& \boldsymbol{\theta}^{R}=\mathbf{r}^{R}\left(\lambda, t^{R}\right) \\
& \lambda_{i}+\theta_{i}^{R} \leq 1
\end{array}
$$

First order conditions for the marginal intervention imply

$\rho \pi_{i}^{R} x_{i} \frac{\partial W}{\partial U_{R}} \frac{\partial u^{R}}{\partial y}+(1-\rho) \pi_{i}^{P} h_{i} \frac{\partial W}{\partial U_{P}}=\mu \Pi_{i} x_{i}$

and $\frac{\partial W}{\partial U_{Z}} \frac{\partial u^{Z}}{\partial y}=\mu$

yielding the solution that for an intervention to be in the statutory package:

$\frac{h_{i}}{x_{i}} \geq \frac{\partial u^{P}}{\partial y}$ evaluated at the post-tax wealth level of the poor.

The policy maker's decision rule now becomes straightforward. The statutory package is chosen by a simple ranking of interventions in line with their cost-effectiveness ratios, with the cut-off rate being the post-tax preferences of the poor. There is no longer any need to consider wealth-related utility losses arising from out-of-pocket payments. The solution differs from the pure 'private insurance' case only in the sense that, through the existence of the statutory package, the poor implicitly receive a tax transfer from the rich, in line with social preferences. The implicit threshold for accepting technologies into the insured package for the poor has therefore been relaxed compared to the purely private case with no such transfers. The rich use the statutory package and secure additional complementary insurance, up to the point where for the marginal intervention:

$\frac{h_{i}}{x_{i}} \geq \frac{\partial u^{R}}{\partial y}$ evaluated at the wealth level after taxes and private insurance have been paid. 
Although the rich wish to use private insurance, their private package is more constrained than under no statutory insurance because the transfer to the poor reduces their wealth, and therefore their willingness to pay for private insurance. The benefit/cost ratio remains the criterion for selecting both statutory and voluntary package, and the system of combined statutory and voluntary insurance replicates a first best solution to health insurance after a socially optimal transfer between wealth groups has been made by the government.

Thus the main role of the statutory package under these circumstances is to effect a financial transfer from rich to poor, allowing the poor access to a broader package of care than would otherwise have been the case. It might at first glance be surprising to see no reference to the epidemiology of diseases in the choice of statutory package, as it is commonly argued that a government concerned with redistribution should concentrate on insuring diseases with high prevalence amongst the poor. Indeed, as we showed earlier, this would be the case if there was sole reliance on public insurance. However, with complementary private insurance it is not necessary. If treatments with relatively high use amongst the rich are included in the statutory package (because they are highly cost-effective), then the associated insurance costs can be recouped in the taxes levied on the rich. Health care payments of the rich comprise (a) an element of tax required to fund their own part of the statutory package, (b) an element of tax required to subsidize the poor's part of the statutory package, and (c) the voluntary insurance premium. Elements (a) and (c) merely reflect in aggregate the costs of their preferred insurance package. The real policy choice is the size of (b), the transfer to the poor.

The solution with voluntary health insurance can be illustrated diagrammatically. Figure 3 shows the health production function for a poor person, constructed by computing the cumulative impact on health of all potential interventions, ranked in decreasing order of benefits:cost ratio. With no subsidy, expenditure $\mathrm{X}_{0}{ }^{\mathrm{P}}$ is chosen. The implicit subsidy from the rich introduced by a statutory insurance package effectively shifts the production function to the left by the amount of the subsidy for each poor person. This leads to a revised expenditure by the poor (which is effectively their tax contribution $t_{P}$ ). Total expected expenditure on health services for the poor is then $\mathrm{X}_{1}{ }^{\mathrm{P}}$, with the tax subsidy from the rich being $\mathrm{X}_{1}{ }^{\mathrm{P}}-t_{P}$. Both utility and health outcome are higher than under no statutory insurance. 
Figure 4 shows the health production function for a rich person. With no subsidy, expenditure $\mathrm{X}_{0}{ }^{\mathrm{R}}$ is chosen. The implicit subsidy to the poor introduced by a statutory insurance package shifts the production function to the right by the amount of the subsidy paid by each rich person. This leads to a revised expenditure by the rich $T_{R}$, comprising the tax contribution $t_{R}$ and any voluntary insurance expenditure. Total expected expenditure on health services for the rich is then $X_{1}{ }^{R}$, with the tax subsidy to the poor being $T_{R}-X_{1}{ }^{R}$. In general, the insurance cover of the rich will comprise a mix of the statutory package and some complementary voluntary coverage. Utility and health outcome are lower than under no statutory insurance.

$<$ Figure 4 about here $>$

For a given statutory package (with voluntary insurance supplement) a rich person can be induced to withdraw entirely from the public coverage if paid a suitable transfer $\hat{y}$. The minimum value of the transfer is such that utility with statutory plus supplementary insurance is equal to utility with purely private insurance plus the transfer, after all taxes and insurance premiums have been paid. In the context of Figure 4, the minimum transfer - a form of compensating variation - is calculated by constructing the indifference curve through the outcome $\left(\mathrm{X}_{1}{ }^{\mathrm{R}}, \mathrm{H}_{1}{ }^{\mathrm{R}}\right)$. The personal production function is then shifted to the right until tangency is secured, the magnitude of the shift indicating the required transfer.

Note that the payment of such transfers would dilute the redistributive role of statutory health insurance. If the rich 'opt out' of the statutory package, the tax base available for the statutory package is reduced. It will usually be the case that the net tax revenue lost by the exit of the richest citizens from the statutory scheme will exceed the reduction in costs associated with their statutory health care expenditure liability. Under these circumstances, there will be no equilibrium social provision, and the statutory package is unviable (Ireland 1990).

\section{Conclusions}

This paper seeks to generalize the formulation and solution of a central problem in all health systems: how to choose the statutory package of care to which all citizens are given free 
access when objectives include financial protection as well as health improvement. A key concern is type of private markets available and the nature of patients' responses when a treatment is not covered by such a package. I have modelled three scenarios: no availability of private care; a spot market of private care paid for out-of-pocket; and a market in prepaid complementary private insurance.

The paper presents optimal decision rules in each case, but notes that that the first two (no private market, or only out-of-pocket private services) may result in a welfare loss compared to a hypothetical first-best solution. In case three, governments can however secure an optimal system of statutory health insurance coverage by specifying a benefits package in line with redistributional goals, and nurturing a complementary voluntary insurance market. Under these circumstances the conventional cost-effectiveness ratio is the appropriate decision rule for including treatments in the package.

In many developing and transition economies there exists no sustainable private insurance market, and citizens must rely on out-of-pocket purchase for many treatments. A policy implication of this analysis is therefore that governments have an important role in stimulating and supporting such a market. Even if this is not possible, welfare improvements can be secured through the specification of a statutory package of care, in line with redistribution and affordability constraints. However, the decision rule for including treatments in such a package must then be amended so as to favour (a) treatments with high incidence amongst the poor and (b) high cost treatments (relative to low cost treatments with identical cost-effectiveness ratios).

A key assumption in this analysis is that treatments purchased in the public and private sectors have identical quality. It is reasonably straightforward to introduce quality variations into the analysis, but it should be noted that they lead to inferior welfare results, and may even make any public insurance unviable if they lead to resistance amongst rich people to funding the statutory package through their taxes. Maintenance of public sector quality is therefore a key policy requirement to make these results applicable.

This analysis reinforces the central role of the economic evaluation of health technologies in the health system, but indicates a possible need to adjust methodology depending on the institutional setting in which it is undertaken. Further work might examine the implications of 
relaxing some of the assumptions in the analysis, such as variable effectiveness of treatment amongst different wealth groups, variable quality, and heterogeneity of health needs within wealth groups.

\section{Declaration}

This paper represents original unpublished work. I am not aware of any conflicts of interest or ethical issues related to its contents. 


\section{References}

Besley, T. (1989). "Publicly provided disaster insurance for health and the control of moral hazard." Journal of Public Economics 39(2): 141-156.

Besley, T. and S. Coate (1991). "Public Provision of Private Goods and the Redistribution of Income." American Economic Review 81(4): 979-984.

Blackorby, C. and D. Donaldson (1988). "Cash versus kind, self-selection and efficient transfers." American Economic Review 78(4): 691-700.

Blomqvist, A. and H. Horn (1984). "Public health insurance and optimal income taxation." Journal of Public Economics 24(3): 353-371.

Blomqvist, A. and P. O. Johansson (1997). "Economic efficiency and mixed public/private insurance." Journal of Public Economics 66(3): 505-516.

Castro-Leal, F., J. Dayton, et al. (2000). "Public spending on health care in Africa: do the poor benefit?" Bulletin of the World Health Organization 78(1): 66-74.

Drummond, M., B. O’Brien, et al. (1997). Methods for the Economic Evaluation of Health Care Programmes. second edition. Oxford, Oxford University Press.

Drummond, M., M. Sculpher, et al. (2005). Methods for the Economic Evaluation of Health Care Programmes, Third Edition. Oxford, Oxford University Press.

Epple, D. and R. Romano (1996). "Ends against the middle: Determining public service provision when there are private alternatives." Journal of Public Economics 62(3): 297-325.

Gertler, P. and J. Van der Gaag (1990). The willingness to pay for medical care in developing countries. Baltimore, Johns Hopkins University Press.

Hauck, K., P. Smith, et al. (2002). The economics of priority setting for health: a literature review. Washington DC, The World Bank.

Hoel, M. (2007). "What should (public) insurance cover?" Journal of Health Economics 26: 251-262.

Ireland, N. (1990). "The mix of social and private provision of goods and services." Journal of Public Economics 43(2): 201-219.

Jost, T., Ed. (2005). Health care coverage determinations. Maidenhead, Open University Press.

McPake, B. (1993). "User charges for health services in developing countries: a review of the economic literature." Social Science \& Medicine 36(11): 1397-1405.

Mossialos, E. and S. Thomson, Eds. (2004). Voluntary health insurance in the European Union. Brussels, World Health Organization.

Munro, A. (1991). "The optimal public provision of private goods." Journal of Public Economics 44(2): 239-261.

Mwabu, G. (2001). User charges for health care: a review of the underlying theory and assumptions. Social Provision in Low-Income Countries - New Patterns and Emerging Trends. G. Mwabu, C. Ugaz and G. White. Oxford, Oxford University Press.

Petretto, A. (1999). "Optimal social health insurance with supplementary private insurance." Journal of Health Economics 18(6): 727-745.

Schreyögg, J., T. Stargardt, et al. (2005). "Defining the 'Health Benefit Basket' in nine European countries." European Journal of Health Economics 6(Suppl 1): 2-10.

Selden, T. M. (1993). "Should the Government Provide Catastrophic Insurance." Journal of Public Economics 51(2): 241-247. 
Smith, P. (2005). "User charges and priority setting in health care: balancing equity and efficiency." Journal of Health Economics 24: 1018-1029.

Smith, P. (2006). Public packages of health care alongside voluntary insurance. Private Voluntary Health Insurance in Development: Friend or Foe? A. Preker, R. M. Scheffler and M. Bassett. Washington DC, World Bank.

Williams, A., A. Tsuchiya, et al. (2005). Eliciting equity-efficiency trade-offs in health. Health policy and economics: opportunities and challenges. P. Smith, L. Ginnelly and M. Sculpher. Maidenhead, Open University Press.

World Health Organization (2001). Macroeconomics and Health: Investing in Health for Economic Development. Report of the Commission on Macroeconomics and Health. Geneva, World Health Organization.

World Health Organization (2002). World Health Report 2002: reducing risks, promoting healthy life. Geneva, World Health Organization. 


\section{Appendix: extension to continuous wealth distribution}

As in the main text, there is a set of $n$ health care problems, and for each problem $i$ there is a technology available at a known constant price $x_{i}$. Wealth is distributed according to the density function $f(y)$. The need for treatment $i$ at wealth level $y$ occurs with probability $\pi_{i}(y)$. Aggregate incidence is given by $\Pi_{i}=\int_{0}^{\infty} \pi_{i}(y) f(y) d y$. The health benefits $h_{i}$ of any treatment are equal for all groups. Taxes for wealth group $y$ are $t(y)$. Utility is separable in wealth and health.

Much of the analysis required with a continuous distribution of wealth requires only a trivial notational change. In the absence of insurance, utility without treatment for the condition represented by $(x, h)$ is $u(y)-h_{i}$ and with treatment is $u\left(y-x_{i}\right)$. There is a critical level of wealth $\xi(x, h)$ at which an individual will be indifferent between receiving and rejecting the treatment, such that $h+u(\xi(x, h)-x)=u(\xi(x, h))$.

With private insurance, patients include treatments in their private package that are not covered publicly, and that satisfy $h / x \geq \partial u / \partial y$ at the post-premium level of wealth.

Following Hoel (2007), assume a government interested in maximizing aggregate utility, possibly weighted by wealth according to weights $w(y)$. With no private health services, the maximization problem is to choose $\lambda$ and the tax function $t(y)$ so as to:

$$
\begin{aligned}
& \text { Maximize } W=\int_{0}^{\infty}\left\{[u(y-t(y))]+\sum_{i} \lambda_{i} h_{i} \pi_{i}(y)\right\} w(y) f(y) d y \\
& \text { Subject to } \sum_{i} \lambda_{i} x_{i} \int_{0}^{\infty} \pi_{i}(y) f(y) d y=\int_{0}^{\infty} t(y) f(y) d y \\
& \lambda_{i} \in\{0,1\}
\end{aligned}
$$

yielding the straightforward result

$$
\frac{h_{i}}{x_{i}} \geq \mu \int_{0}^{\infty} \pi_{i}(y) f(y) d y / \int_{0}^{\infty} \pi_{i}(y) w(y) f(y) d y .
$$

That is, treatments are selected if their benefit:cost ratio exceeds the threshold $\mu$, possibly weighted for equity. Tax contributions satisfy the marginal conditions $\partial u / \partial y=\mu / w(y)$ 
With complementary out-of-pocket health care, and ignoring the equity term $w($.$) , the$ government's welfare maximization problem can be written as:

$$
\begin{aligned}
\text { Maximize } & W=\sum_{i} \lambda_{i} \int_{0}^{\infty}[u(y-t(y))] \pi_{i}(y) f(y) d y+\sum_{i}\left(1-\lambda_{i}\right) \int_{0}^{\xi\left(x_{i}, h_{i}\right)}\left[u(y-t(y))-h_{i}\right] \pi_{i}(y) f(y) d y \\
& +\sum_{i}\left(1-\lambda_{i}\right) \int_{\xi\left(x_{i}, h_{i}\right)}^{\infty}\left[u\left(y-t(y)-x_{i}\right)\right] \pi_{i}(y) f(y) d y
\end{aligned}
$$

Subject to $\sum_{i} \lambda_{i} x_{i} \int_{0}^{\infty} \pi_{i}(y) f(y) d y=\int_{0}^{\infty} t(y) f(y) d y$

$$
\lambda_{i} \in\{0,1\}
$$

The three terms in the maximand indicate, respectively, the benefits to all citizens arising from inclusion in the statutory package, the health loss to poorer people arising from exclusion from the package, and the wealth loss to richer people arising from exclusion (Hoel, 2007). This formulation gives rise to the solution:

$$
\int_{0}^{\xi\left(x_{i}, h_{i}\right)} h_{i} \pi_{i}(y) f(y) d y+\int_{\xi\left(x_{i}, h_{i}\right)}^{\infty}\left[u(y-t(y))-u\left(y-t(y)-x_{i}\right)\right] \pi_{i}(y) f(y) d y \geq \mu x_{i} \Pi_{i}
$$

Compared with conventional cost-effectiveness analysis, this indicates that the benefits of including a treatment in the statutory package must be amended for wealth-related utility loss amongst richer people, which will be less than health gains $h_{i}$ for that group. This rule implicitly (a) reduces the weight placed on richer people, so will favour diseases that are more prevalent amongst the poor, and (b) increases the weight placed on more expensive treatments, for which the value of $\xi(x, h)$ is low. Inclusion of equity weights is a trivial extension.

When there exists the possibility of complementary private insurance a continuous distribution of incomes, there is a critical level of wealth $\varsigma(x, h)$ for each treatment above which the treatment will be included in the insurance package. The optimization problem now becomes: 
Maximize $W=\int_{0}^{\infty}[u(y-t(y)-p(y))] f(y) d y-\sum_{i}\left(1-\lambda_{i}\right) \int_{0}^{\varsigma\left(x_{i}, h_{i}\right)} h_{i} \pi_{i}(y) f(y) d y$

$$
\text { where } p(y)=\sum_{\frac{h_{i}}{x_{i}} \frac{\partial u}{\partial y}}\left(1-\lambda_{i}\right) \pi_{i}(y) x_{i}
$$

Subject to $\sum_{i} \lambda_{i} x_{i} \int_{0}^{\infty} \pi_{i}(y) f(y) d y=\int_{0}^{\infty} t(y) f(y) d y$

$$
\lambda_{i} \in\{0,1\}
$$

yielding the solution

$$
\int_{\varsigma\left(x_{i}, h_{i}\right)}^{\infty} \frac{\partial u}{\partial y} \pi_{i}(y) x_{i} f(y) d y+\int_{0}^{\varsigma\left(x_{i}, h_{i}\right)} h_{i} \pi_{i}(y) f(y) d y \geq \mu \Pi_{i} x_{i}
$$

which reduces to $\int_{0}^{\varsigma\left(x_{i}, h_{i}\right)} h_{i} \pi_{i}(y) f(y) d y \geq \mu x_{i}\left(\Pi_{i}-\int_{\varsigma\left(x_{i}, h_{i}\right)}^{\infty} \pi_{i}(y) f(y) d y\right)=\mu x_{i} \int_{0}^{\varsigma\left(x_{i}, h_{i}\right)} \pi_{i}(y) f(y) d y$ or $h_{i} / x_{i} \geq \mu$ 


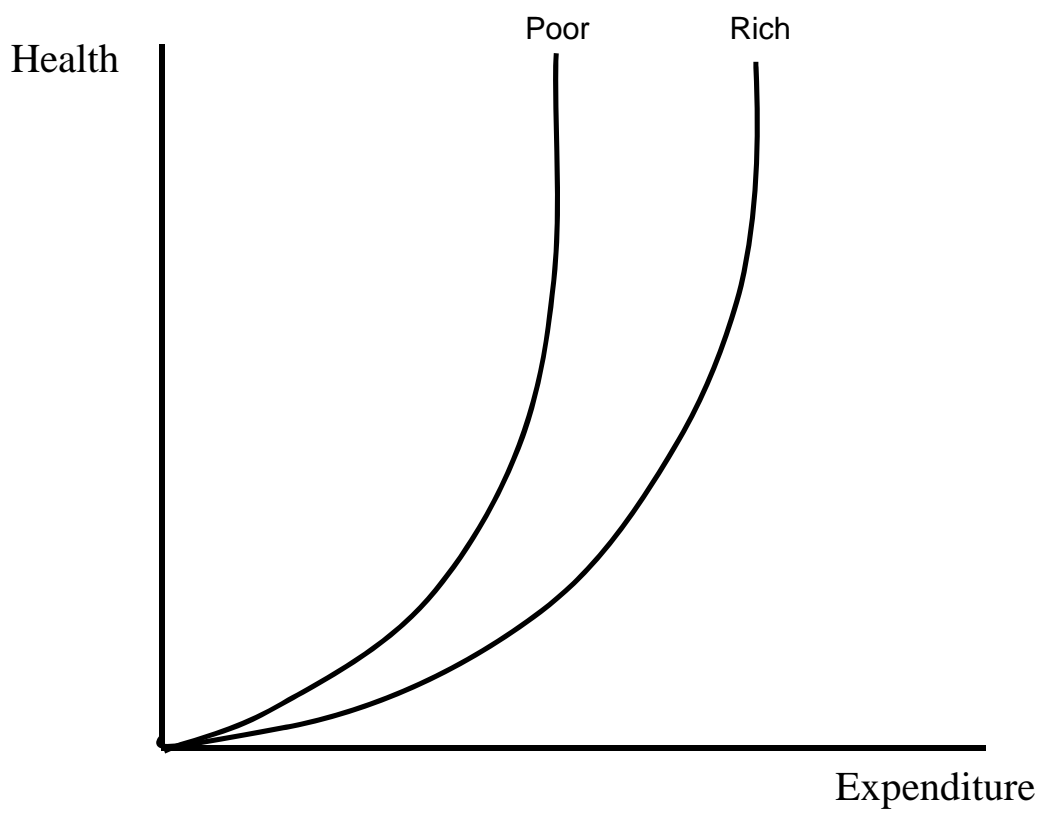

Figure 1: Indifference between treatment and non-treatment for rich and poor

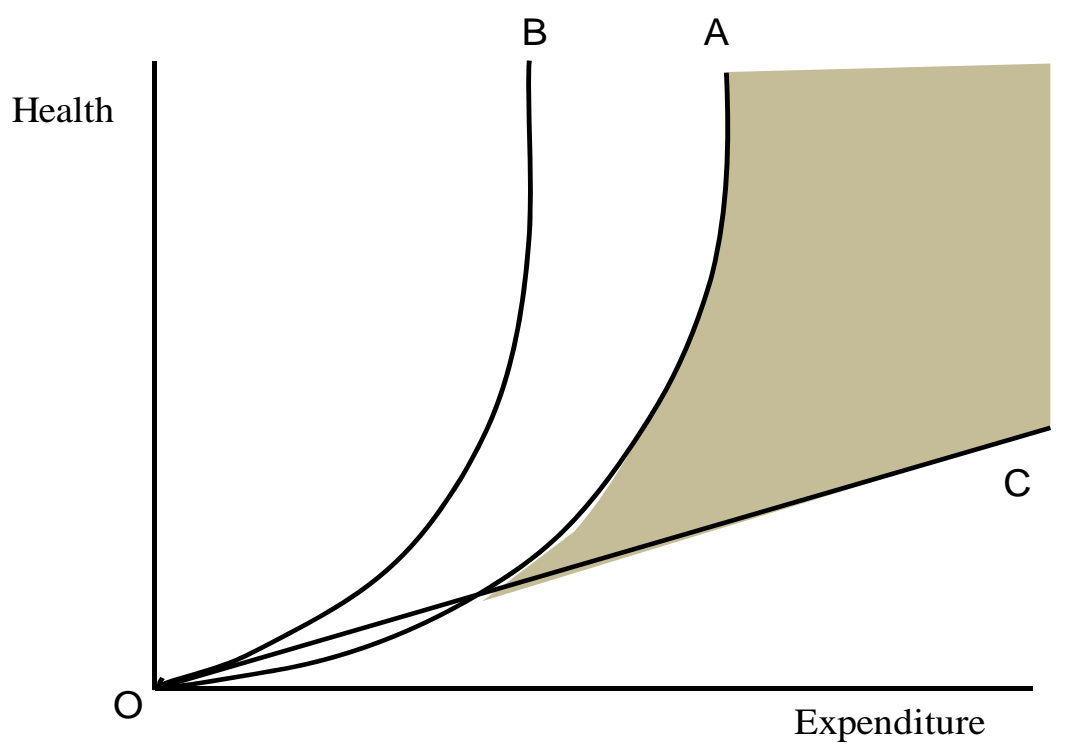

Figure 2: Impact of health insurance on access to treatments 


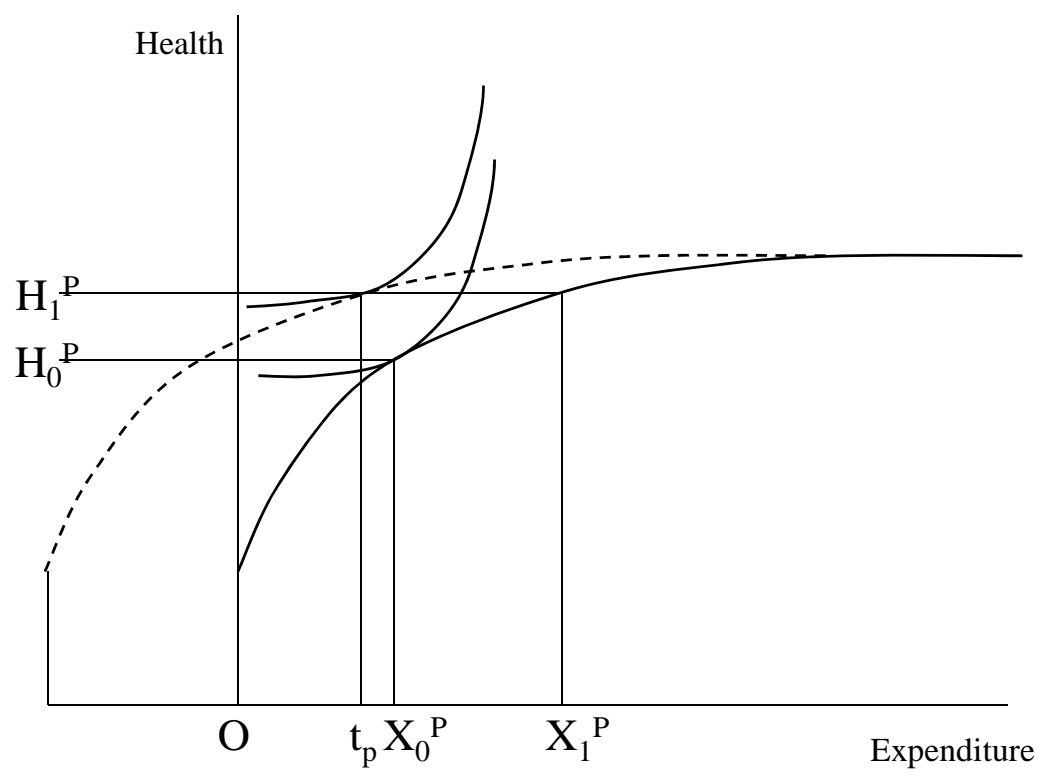

Figure 3: Health expenditure by the poor with introduction of a statutory package

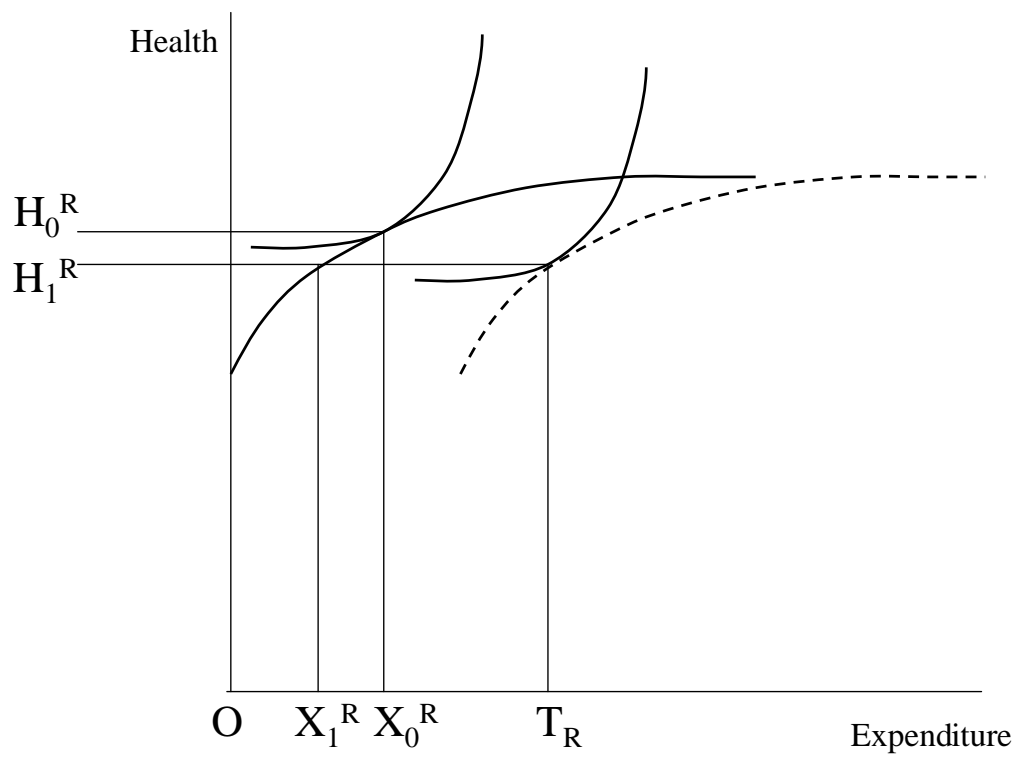

Figure 4: Health expenditure by the rich with a statutory package 